\title{
COSMOGRAIL: the COSmological MOnitoring of GRAvltational Lenses
}

\section{SDSS J0924+0219: the redshift of the lensing galaxy, the quasar spectral variability and the Einstein rings ${ }^{\star}$}

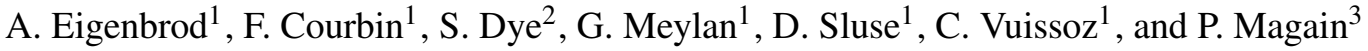 \\ ${ }^{1}$ Laboratoire d'Astrophysique, École Polytechnique Fédérale de Lausanne (EPFL), Observatoire, 1290 Sauverny, Switzerland \\ e-mail: alexander.eigenbrod@epfl.ch \\ 2 School of Physics and Astronomy, Cardiff University, 5 The Parade, Cardiff, CF24 3YB, UK \\ ${ }^{3}$ Institut d'Astrophysique et de Géophysique, Université de Liège, Allée du 6 août 17, Sart-Tilman, Bât. B5C, 4000 Liège, Belgium
}

Received 26 October 2005 / Accepted 7 February 2006

\begin{abstract}
Aims. To provide the observational constraints required to use the gravitationally lensed quasar SDSS J0924+0219 for the determination of $H_{0}$ from the time delay method. We measure here the redshift of the lensing galaxy, we show the spectral variability of the source, and we resolve the lensed host galaxy of the source.

Methods. We present our VLT/FORS1 deep spectroscopic observations of the lensed quasar SDSS J0924+0219, as well as archival HST/NICMOS and ACS images of the same object. The two-epoch spectra, obtained in the Multi Object Spectroscopy (MOS) mode, allow for very accurate flux calibration and spatial deconvolution. This strategy provides spectra for the lensing galaxy and for the quasar images A and B, free of any mutual light contamination. We deconvolve the HST images as well, which reveal a double Einstein ring. The mass distributions in the lens, reconstructed in several ways, are compared.

Results. We determine the redshift of the lensing galaxy in SDSS J0924+0219: $z_{\text {lens }}=0.394 \pm 0.001$. Only slight spectral variability is seen in the continuum of quasar images A and B, while the C III], Mg II and Fe II emission lines display obvious changes. The flux ratio between the quasar images $\mathrm{A}$ and $\mathrm{B}$ is the same in the emission lines and in the continuum. One of the Einstein rings found using deconvolution corresponds to the lensed quasar host galaxy at $z=1.524$ and a second bluer one, is the image either of a star-forming region in the host galaxy, or of another unrelated lower redshift object. A broad range of lens models give a satisfactory fit to the data. However, they predict very different time delays, making SDSS J0924+0219 an object of particular interest for photometric monitoring. In addition, the lens models reconstructed using exclusively the constraints from the Einstein rings, or using exclusively the astrometry of the quasar images, are not compatible. This suggests that multipole-like structures play an important role in SDSS J0924+0219.
\end{abstract}

Key words. gravitational lensing - cosmology: cosmological parameters - quasars: individual: SDSSJ0924+0219 - dark matter

\section{Introduction}

COSMOGRAIL is a multi-site optical monitoring campaign of lensed quasars. Following the original work by Refsdal (1964), its goal is to measure, with an accuracy close to one percent (Eigenbrod al. 2005), the so-called time delay between the images of most gravitationally lensed quasars. These time delays are used in combination with lens models and detailed observations of individual systems to infer the value of the Hubble parameter $H_{0}$, independent of any standard candle (e.g., reviews by Courbin et al. 2002; Kochanek 2005a).

The present work is devoted to the quadruply imaged quasar SDSS J0924+0219 (Inada et al. 2003) at $z=1.524$, discovered

\footnotetext{
* Based on observations made with the ESO-VLT Unit Telescope 2 Kueyen (Cerro Paranal, Chile; Program 074.A-0563, PI: G. Meylan) and on data obtained with the NASA/ESA Hubble Space Telescope (Program HST-GO-9744, PI: C. S. Kochanek) and extracted from the data archives at the Space Telescope Science Institute, which is operated by the Association of Universities for Research in Astronomy, Inc., under NASA contract NAS 5-26555.
}

in the course of the Sloan Digital Sky Survey (SDSS). This object is particularly interesting because of its anomalous image flux ratios, the origin of which is unclear. It has been argued that the faintest image of SDSS J0924+0219, which is located at a saddle point of the arrival-time surface, could be demagnified either from star microlensing (Schechter et al. 2004; Keeton et al. 2006) or subhalos microlensing (Kochanek \& Dalal 2004).

We analyse here our deep optical spectra of SDSS J0924+0219 obtained with the ESO Very Large Telescope (VLT). These spectra are used to: 1- measure the redshift of the lensing galaxy; 2 - estimate the spectral variability of the quasar; 3- measure the flux ratio between images A and B of SDSS J0924+0219, in the continuum and the broad emission lines. Hubble Space Telescope (HST) ACS and NICMOS images from the STScI archives are deconvolved using the MCS algorithm (Magain et al. 1998) which unveils two Einstein rings. One of the rings corresponds to the host galaxy of the quasar source and is used to constrain the lens models. The second one 


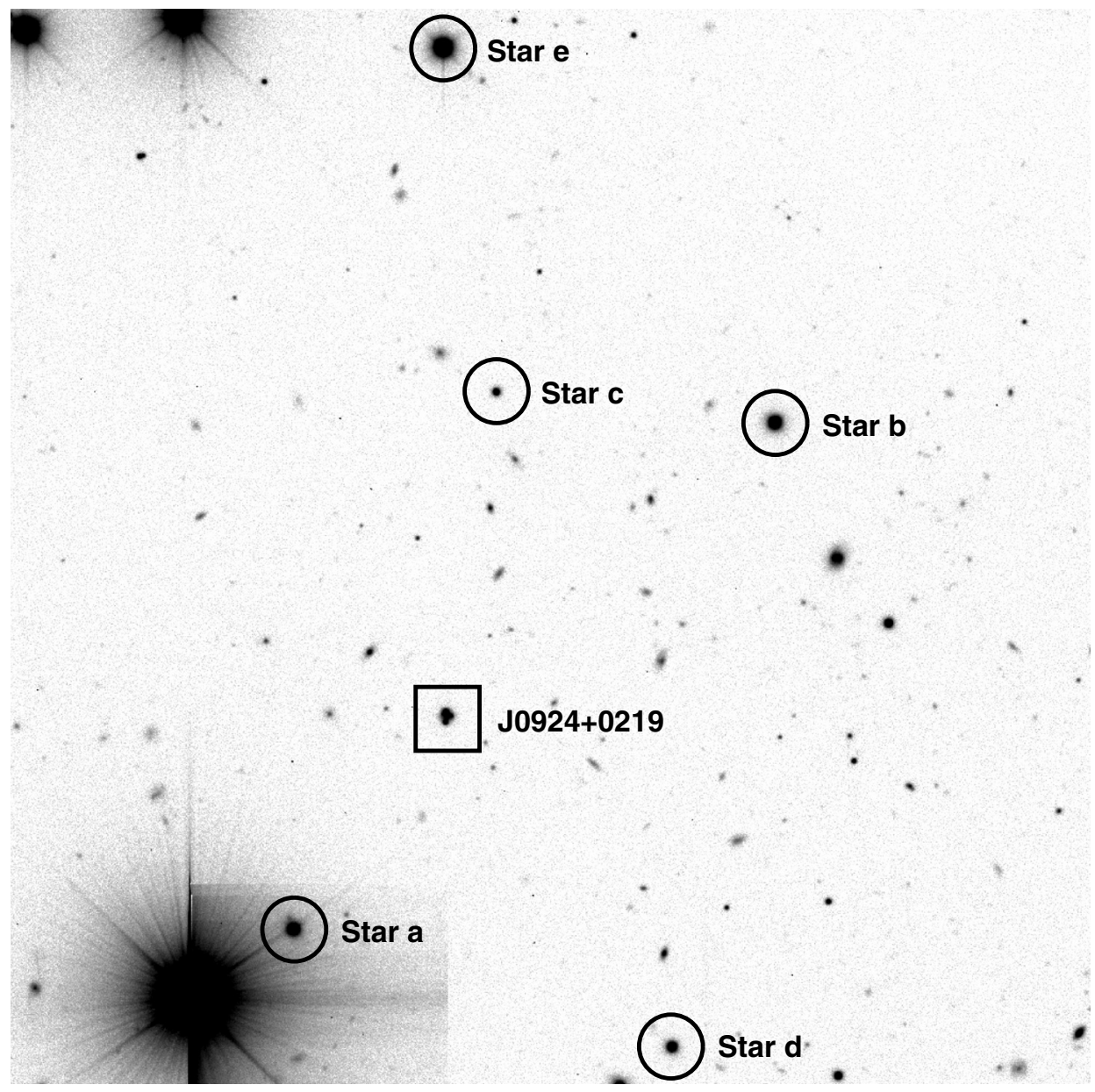

Fig. 1. $R$-band VLT image of SDSS J0924+0219, where objects are labeled following Inada et al. (2003). The stars a, c, d, and e are used to compute the PSF spectrum (see text). Only stars a, $d$ and e are used to derive the relative flux calibration between each MOS mask. The field of view is $3.4^{\prime} \times 3.4^{\prime}$, North is to the top, East to the left.

is probably due to a star-forming region in the host galaxy of the quasar source or to another unrelated object.

\section{VLT spectroscopy}

\subsection{Observations}

Our spectroscopic observations of SDSS J0924+0219 are part of a low dispersion spectroscopic survey aimed at measuring all unknown lens redshifts. They are acquired with the FOcal Reducer and low dispersion Spectrograph (FORS1), mounted on the ESO Very Large Telescope, used the MOS mode (Multi Object Spectroscopy) and the high resolution collimator. This configuration allows the simultaneous observation of a total of 8 objects over a field of view of $3.4^{\prime} \times 3.4^{\prime}$ with a pixel scale of $0.1^{\prime \prime}$ (Fig. 1). The G300V grism, used in combination with the GG435 order sorting filter, leads to the useful wavelength range $4450 \AA<\lambda<8650 \AA$ and to a scale of $2.69 \AA$ per pixel in the spectral direction. This setup has a spectral resolution $R=\lambda / \Delta \lambda \simeq 300$ at the central wavelength $\lambda=5900 \AA$, which translates in velocity space to $\Delta v=\mathrm{c} \Delta \lambda / \lambda \simeq 1000 \mathrm{~km} \mathrm{~s}^{-1}$.

The slitlets of the MOS mask are all 19" long and 0.7" wide, which both avoids lateral contamination by the quasar image $\mathrm{C}$ and matches well the seeing values during the observations. Four slits were centered on the foreground stars a, c, d, e, while a fifth slit is centered on images A and B of SDSS J0924+0219, after rotation of the mask to a suitable Position Angle (PA) (Fig. 2).

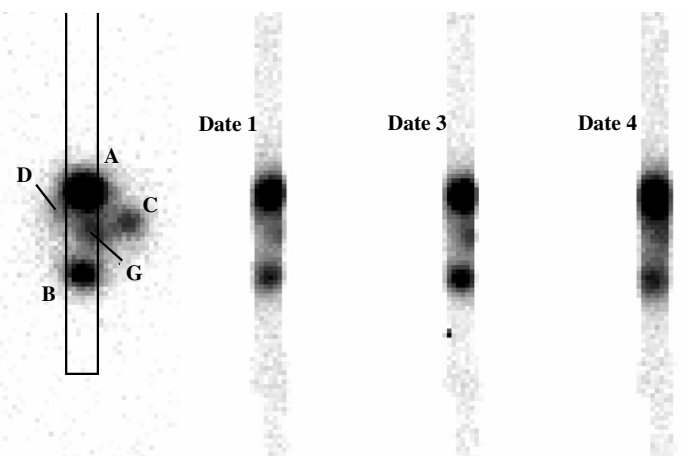

Fig. 2. $R$-band images of SDSS J0924+0219. A short $30 \mathrm{~s}$ exposure is shown on the left, where the quasar images A, B, C and D as well as the lensing galaxy, are indicated. The seeing is $0.37^{\prime \prime}$ in this image and the pixel scale is $0.10^{\prime \prime}$. The position of the $0.70^{\prime \prime}$ slitlets is also indicated. They correspond to three epochs of observations with very different seeings (see Table 1). The slit has not moved at all between the exposures, even when taken 15 days apart.

The spectra of the stars are used both to compute the reference Point Spread Function (PSF) needed for the deconvolution and to carry out a very accurate relative flux calibration. "Throughslit" images acquired just before exposures \#1, \#3, \#4 in order to check the mask alignment are displayed in Fig. 2. 


\subsection{Reduction and deconvolution}

The spectra are bias subtracted and flat-fielded using IRAF ${ }^{1}$. The flat fields for each slitlet are created from 5 dome exposures, using cosmic ray rejection. They are normalized by averaging 60 lines along the spatial direction, rejecting the 20 highest and 20 lowest pixels, then block replicating the result to match the physical size of the individual flat fields.

Wavelength calibration is obtained from numerous emission lines in the spectrum of Helium-Argon lamps. The wavelength solution is fitted in two dimension to each slitlet of the MOS mask. The fit uses a fifth-order Chebyshev polynomial along the spectral direction and a third-order Chebyshev polynomial fit along the spatial direction. Each spectrum is interpolated following this fit, using a cubic interpolation. This procedure ensures that the sky lines are well aligned with the columns of the CCD after wavelength calibration. The wavelength solution with respect to the reference lines is found to be very good, with an rms scatter better than $0.2 \AA$ for all spectra.

The sky background is then removed by fitting a secondorder Chebyshev polynomial in the spatial direction to the areas of the spectrum that are not illuminated by the object.

Finally, we perform the cosmic ray removal as follows. First, we shift the spectra in order to align them spatially (this shift is only a few tenths of a pixel). Second, we create a combined spectrum for each object from the 6 exposures, removing the 2 lower and 2 higher pixels, after applying appropriate flux scaling. The combined spectrum obtained in that way is cosmic ray cleaned and used as a reference template to clean the individual spectra. We always check that neither the variable seeing, nor the variability of the quasar causes artificial loss of data pixels.

Even though the seeing on most spectra is good, the lensing galaxy is close enough to the brightest quasar images $\mathrm{A}$ and $\mathrm{B}$ to be affected by significant contamination from the wings of the PSF. For this reason, the spectral version of MCS deconvolution algorithm (Magain et al. 1998; Courbin et al. 2000) is used in order to separate the spectrum of the lensing galaxy from the spectra of the quasar images. The MCS algorithm uses the spatial information contained in the spectrum of a reference PSF, which is obtained from the slitlets positioned on the four isolated stars a, c, d, and e (Fig. 1). The final normalized PSF is a combination of the four PSF spectra. The six individual spectra are deconvolved separately, extracted, flux calibrated as explained in Sect. 2.3 and combined. The spectrum of the lensing galaxy is extracted from the "extended channel" of the deconvolved data, while the spectra of the quasar images are extracted from the "point-source channel" (see Courbin et al. 2000).

\subsection{Flux calibration}

Our absolute flux calibration is based on the spectrum of the spectrophotometric standard star Feige 66 taken on the night of 2005 January 16 . The response function of the grism is determined for this single epoch. It is cross calibrated using stars observed in each MOS mask in order to obtain a very accurate calibration across all epochs. The spectra of four stars are displayed in Fig. 3, without any deconvolution and having used a $4^{\prime \prime}$ aperture for extraction. We find significant differences in flux between the six epochs, that need to be corrected for. The

${ }^{1}$ IRAF is distributed by the National Optical Astronomy Observatories, which are operated by the Association of Universities for Research in Astronomy, Inc., under cooperative agreement with the National Science Foundation.

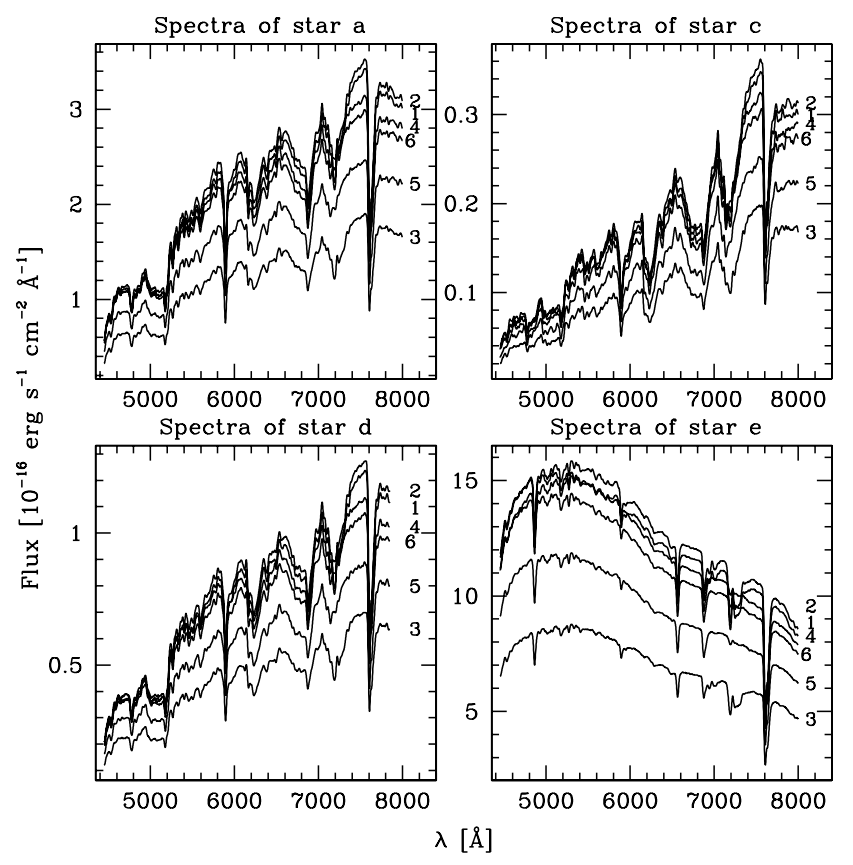

Fig. 3. The spectra of the foreground stars. The index on the right of each spectrum indicates the exposure number, following Table 1. Flux differences are mainly due to the presence of light clouds on observation dates \#3, \#5 and \#6.

Table 1. Journal of the VLT spectroscopic observations of SDSS J0924+0219. The seeing is measured on the spectrum of the PSF stars. The exposure time is $1400 \mathrm{~s}$ for each of the 6 epochs.

\begin{tabular}{ccccc}
\hline \hline ID & Date & Seeing ["] & Airmass & Weather \\
\hline 1 & $14 / 01 / 2005$ & 0.66 & 1.188 & Photometric \\
2 & $14 / 01 / 2005$ & 0.59 & 1.150 & Photometric \\
3 & $14 / 01 / 2005$ & 0.46 & 1.124 & Light clouds \\
4 & $01 / 02 / 2005$ & 0.83 & 1.181 & Photometric \\
5 & $01 / 02 / 2005$ & 0.97 & 1.146 & Light clouds \\
6 & $01 / 02 / 2005$ & 0.84 & 1.126 & Light clouds \\
\hline
\end{tabular}

main causes for these differences are variable seeing and variable extinction due to thin cirrus during some of the observations (Table 1). The effect of mask misalignment is excluded, as can be seen from the image-through-slit of Fig. 2.

Assuming that the intrinsic flux of the foreground stars has not varied between the six exposures, and taking the data \#1 of Table 1 as a reference, we derive the flux ratio between this reference epoch and the six other dates, for each star. These curves, fitted with a third-order polynomial, are shown in Fig. 4. The corrections computed in this way are found to be very stable across the mask: the curves obtained for two different stars only showed slight oscillations with an amplitude below $2 \%$. This is also the accuracy of the flux correction between different epochs. A mean correction curve is then computed for each epoch from all stars, except star c which is much fainter than the others, and is applied to the deconvolved spectra of the quasars and of the lensing galaxy.

\section{Extracted spectra}

\subsection{The lensing Galaxy}

The six deconvolved spectra of the lensing galaxy are extracted, combined, and smoothed with a $5 \AA$ box ( 2 pixels). Figure 5 shows the final one-dimensional spectrum, where the $\mathrm{Ca}$ II $\mathrm{H}$ $\& \mathrm{~K}$ absorption lines are obvious, as well as the $4000 \AA$ break, 


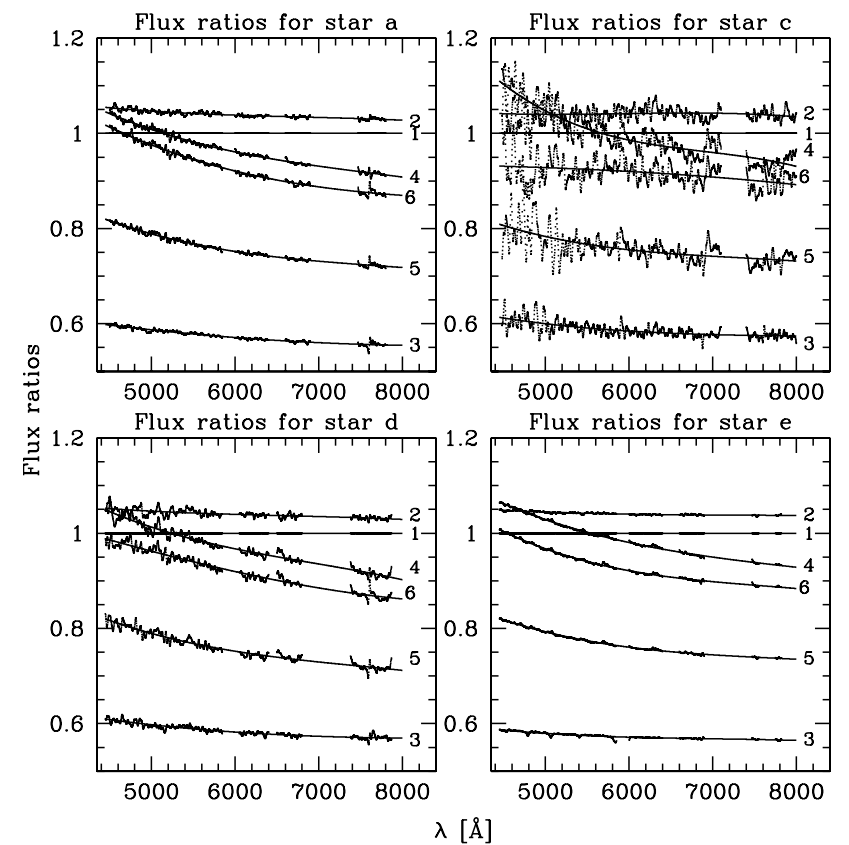

Fig. 4. Flux ratios between Date \#1 and the 5 others, along with the third-order polynomial fits. We use the ratios of the 3 stars: $a$, $d$ and e to determine the mean correction applied to the quasar. Star c, which is much fainter than the others, is excluded from the final calibration. The (small) parts of the spectra with strong atmospheric absorption are masked during the polynomial fit. The peak-to-peak differences between the ratios computed using star a, $\mathrm{d}$ and e, differ by less than $2 \%$.

the G-band typical for $\mathrm{CH}$ absorption, the $\mathrm{Mg}$ band, and the $\mathrm{H} \beta$, and Fe II absorption lines. These features yield a mean redshift of $z_{\text {lens }}=0.394 \pm 0.001$, where the $1-\sigma$ error is the standard deviation between all the measurements on the individual lines, divided by the square root of the number of lines used. We do not consider the $4000 \AA$ A break in these calculations. This spectroscopic redshift falls very close to the photometric estimate of $z=0.4$ by Inada et al. (2003), and agrees with the spectroscopic redshift of Ofek et al. (2005). In addition, the absence of emission lines confirms a gas-poor early-type galaxy. No trace of the quasar broad emission lines is seen in the spectrum of the lensing galaxy, indicative of an accurate decomposition of the data into the extended (lens) and point source (quasar images) channels.

\subsection{The quasar images}

The mean spectra of quasar images A and B are shown in Fig. 6, smoothed with a $5 \AA$ box. The Al III], Si III], C III], [Ne IV] and Mg II broad emission lines are clearly identified. A Gaussian fit to these 5 lines yield a mean redshift of $1.524 \pm 0.001$ for image A and $1.524 \pm 0.002$ for the fainter image B. The standard deviation between the fits to the individual lines, divided by the square root of the number of lines used, is taken as the error bar. These results are in excellent agreement with the values obtained by Inada et al. (2003), as well as the redshift from the SDSS database, who both report $z=1.524$.

\subsection{Variability of the quasar images}

The spectra of quasar images A and B are shown in Fig. 7 for 2005 January 14 and February 1. These are the mean of

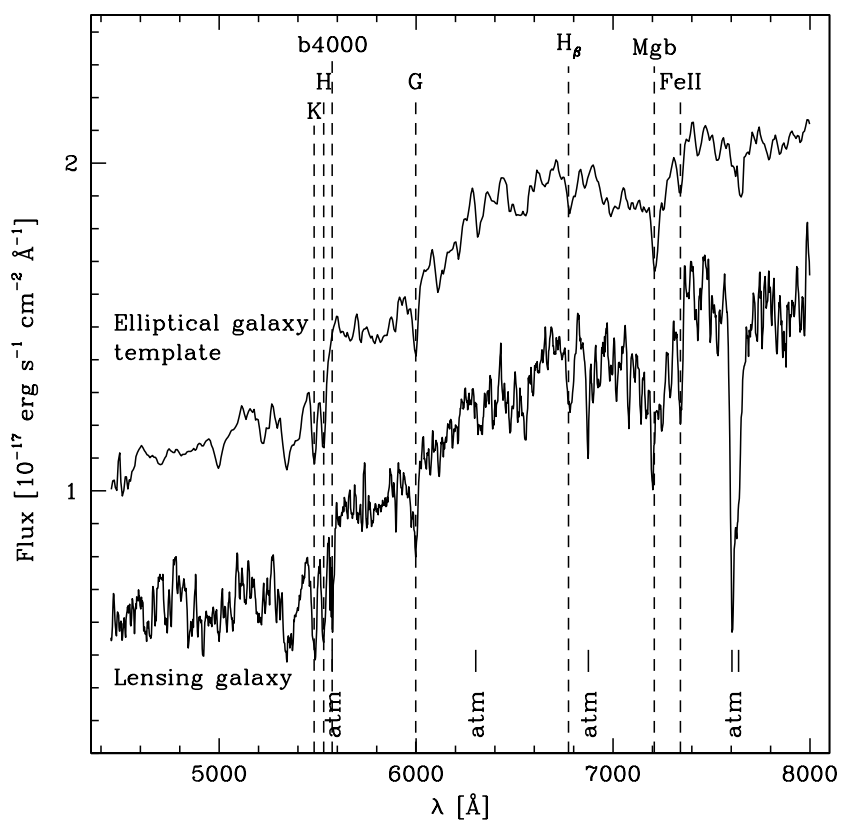

Fig. 5. Spectrum of the lensing galaxy in SDSS J0924+0219, as obtained by combining the data for the 6 epochs, i.e., a total integration time of $8400 \mathrm{~s}$. The template spectrum of an elliptical galaxy at $z=0.394$ is also shown for comparison (Kinney et al. 1996). All main stellar absorption lines are well identified. Prospects for a future determination of the galaxy's velocity dispersion are therefore excellent.

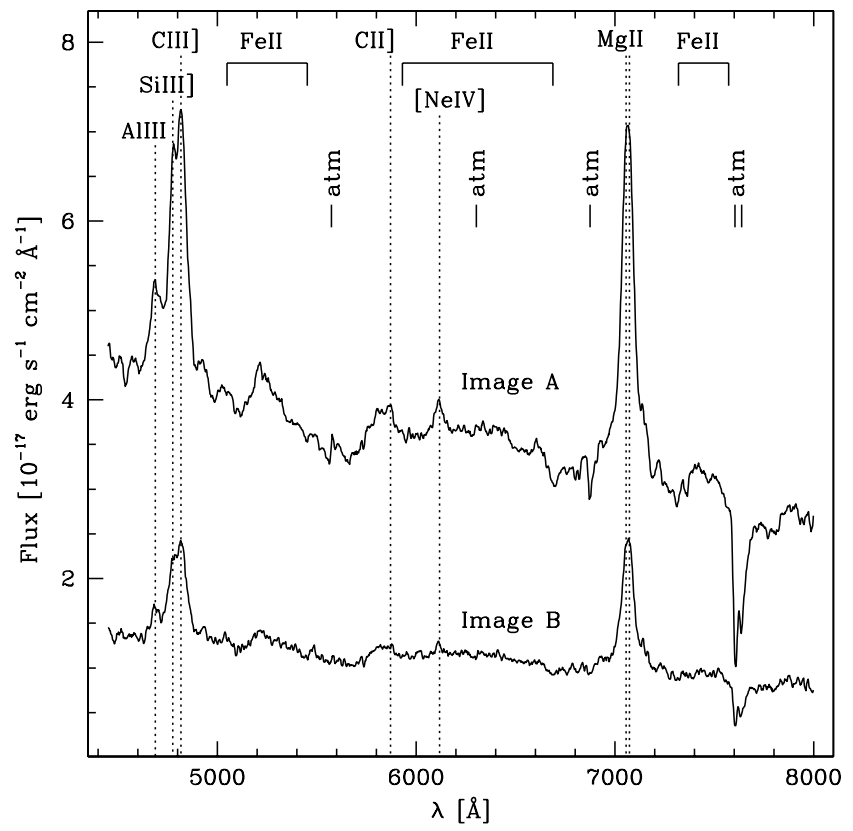

Fig. 6. Spectra of the quasar images A and B of SDSS J0924+0219, as extracted from the deconvolved data. These figure shows the mean of the 6 spectra taken for the 6 epochs, after the flux calibration described in Sect. 2.3.

the three spectra obtained on each date, smoothed with a $5 \AA$ box. Although the continuum shows little variation (only B has fadded slightly between our two observing dates), there are obvious changes in the broad emission lines of each quasar image. In image $\mathrm{A}$, the red wing of the $\mathrm{Mg}$ II emission line has brightened, as well as the $\mathrm{CII}$ ] emission line, while in image $\mathrm{B}$, the center of the C III] emission line has become double peaked and the Fe II feature redwards of Mg II has fadded. A zoom on these lines 


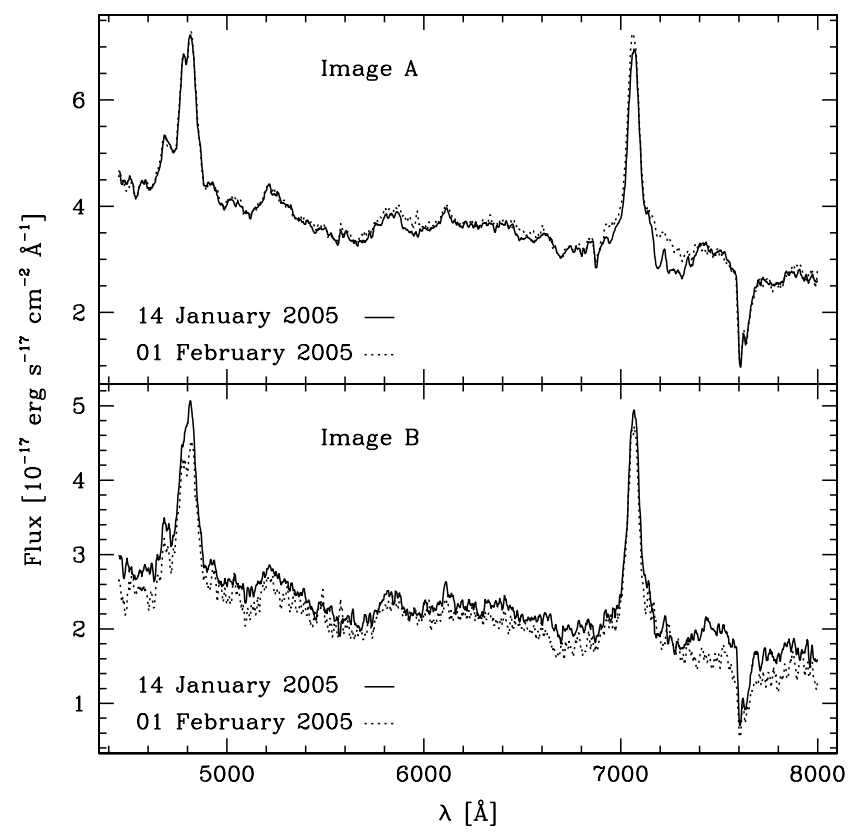

Fig. 7. The spectra of images A and B on 14 January and 1 February 2005 show a stable continuum for both images, but the broad emission lines do vary on a time-scale of two weeks (see Fig. 8).

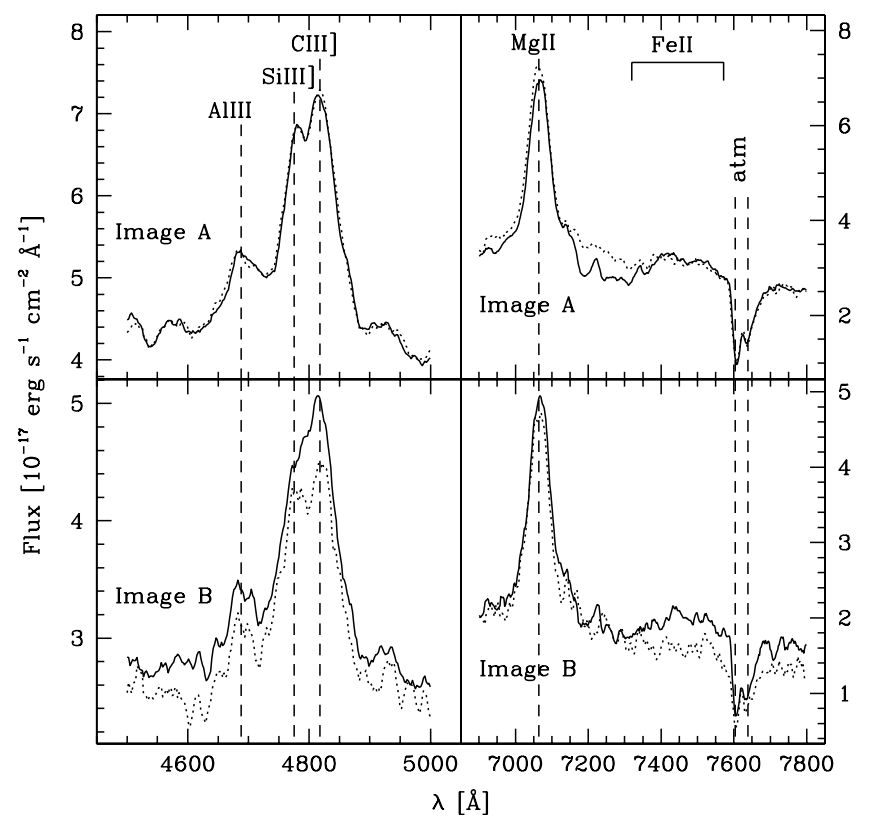

Fig. 8. Enlargements of Fig. 7 comparing the broad emission lines of images A and B on 14 January (solid curve) and 1 February 2005 (dotted curve). Obvious variations are seen in the red wing of the $\mathrm{Mg}$ II in image $\mathrm{A}$, in the center of the $\mathrm{C} \mathrm{III]}$ in image $\mathrm{B}$. The Fe II feature redwards of $\mathrm{Mg}$ II in image B has also changed by $20 \%$. These variations are asymmetric about the center of the lines. The asymmetry is different in $\mathrm{C} \mathrm{III]} \mathrm{and} \mathrm{Mg}$ II.

is shown in Fig. 8. The line variations are already visible before averaging the 3 individual spectra at a given date and in the not-so-blended quasar images of the raw un-deconvolved spectra. We can therefore safely rule out any deconvolution artefacts due to PSF variations in the MOS mask. In addition, the residual images after deconvolution (see Courbin et al. 2000, for more details) are particularly good, indicative of little or no PSF variations across the slitlet mask.

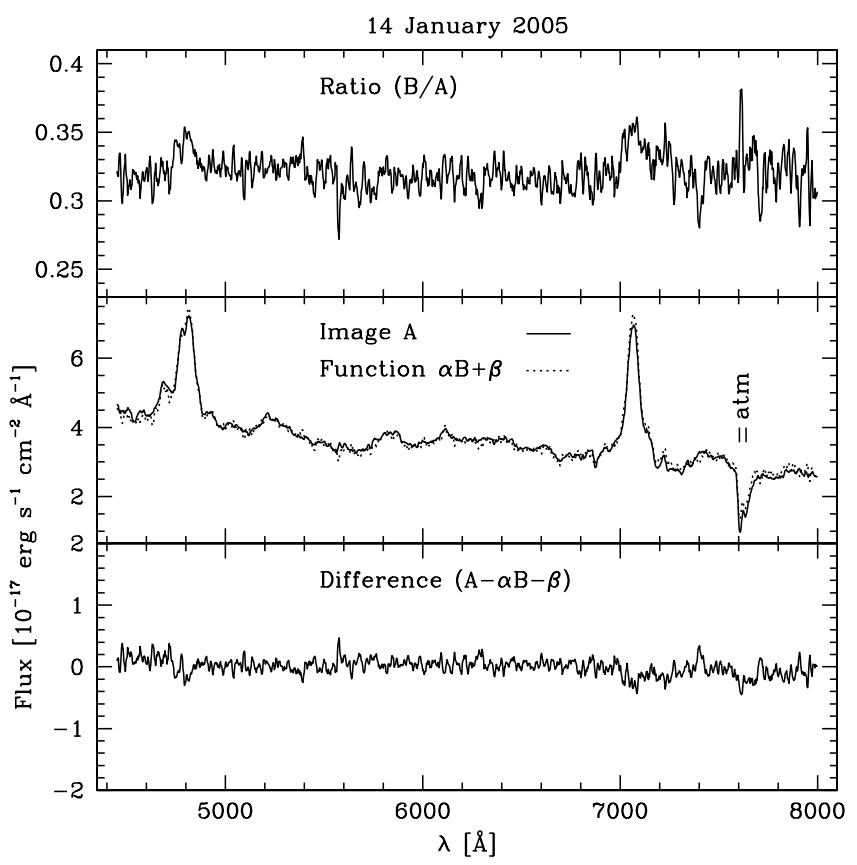

Fig. 9. Comparison between the spectra of images A and B taken on 14 January 2005. The top panel shows the dimensionless ratio B/A. The mean ratio is 0.32 . In the middle panel, a first-order polynomial $\alpha \mathrm{B}+\beta$ is fit to the spectra of image $\mathrm{A}$. The best fit is obtained with $\alpha=2.80 \pm 0.05$ and $\beta=0.37$. The difference in flux between A and the fitted $\alpha \mathrm{B}+\beta$ polynomial is displayed in the bottom panel, and does not exceed a few percent of the flux.

\subsection{Image flux ratio}

Keeton et al. (2006) have recently observed that the flux ratio between the images of SDSS J0924+0219 is different in the continuum and in the broad emission lines. In their slitless HST/ACS observations, the flux ratio between A and B is 2.60 in the emission lines, and about 3.5 in the continuum, i.e., the emission lines are $30 \%$ different from the continuum.

We plot the flux ratio between quasar image B and A as a function of wavelength at a given date (top panels in Figs. 9 and 10). This ratio is close to flat, with some small differences in the broad emission lines.

We construct the spectrum $\alpha \mathrm{B}+\beta$ and adjust the parameters using a linear least squares fit so that it matches the spectrum of quasar A. The result is shown in the middle panels of Figs. 9 and 10. Almost no trace of the emission lines are seen in the difference spectra in the bottom panels of the figure. Our spectra indicate no strong differential amplification of the continuum and broad emission lines in the components $\mathrm{A}$ and $\mathrm{B}$ of SDSS J0924+0219, and the small residual seen in the emission lines in the bottom panels of Figs. 9 and 10 are an order of magnitude smaller than reported in Keeton et al. (2006).

In the 15 days separating the observations, $\alpha$ has changed by only $2 \%$. For both dates the residuals of the fit are almost perfectly flat, indicating no continuum change. Only asymmetric changes in the emission lines are seen.

Finally, the flat flux ratio between image $\mathrm{A}$ and $\mathrm{B}$ shows that there is no significant extinction by interstellar dust in the lensing galaxy. 


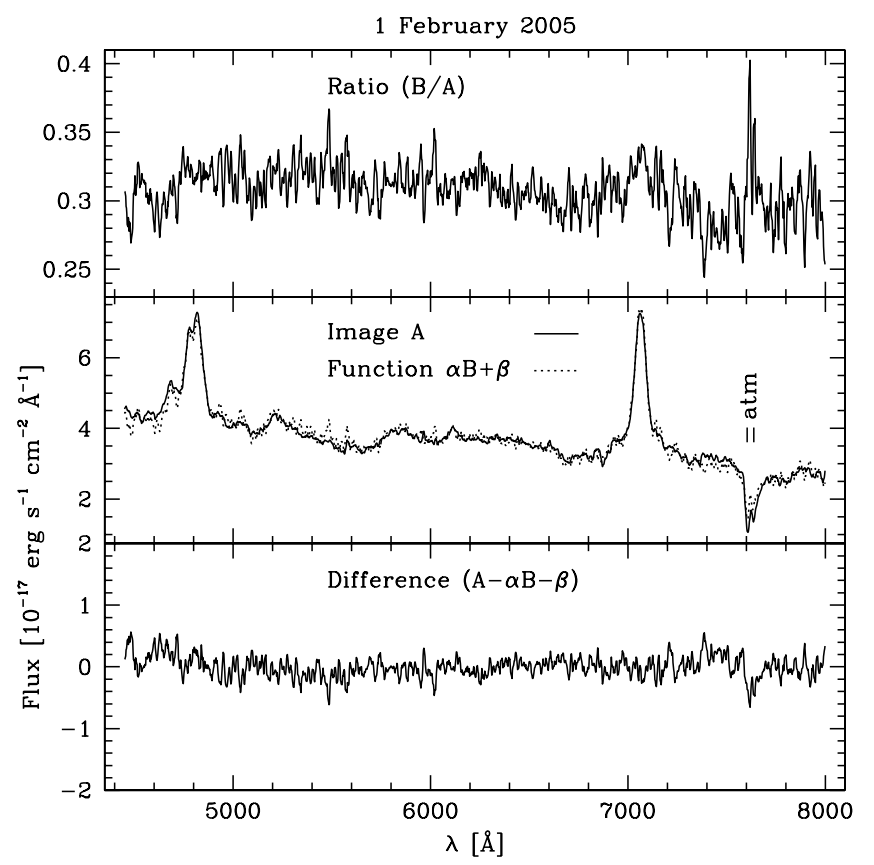

Fig. 10. Same as in Fig. 9 but for the spectra taken on 1 February 2005. The mean $\mathrm{B} / \mathrm{A}$ ratio is 0.31 , and the best fit of image $\mathrm{A}$ is obtained with $\alpha=2.86 \pm 0.05$ and $\beta=0.43$.

\subsection{Intrinsic variations vs. microlensing}

It is hard, with only two observing points, to infer the origin of the spectral variations observed in SDSS J0924+0219. Nevertheless, we see rapid (15 days) and asymmetric changes in the emission lines of the quasar images, and no strong changes in the continuum. Intrinsic variations of quasars are usually stronger in the continuum than in the emission lines, and they are also longer than the two-week span we observe here. Such rapid variations due to microlensing have been seen in at least one other lensed quasar: HE 1104-1805 (Schechter et al. 2003). SDSS J0924+0219 might be a second such case. Microlensing variability is supported by the photometric broad-band data by Kochanek et al. (2005b), showing that A and B have very different light curves that are hard to match even after shifting them by the expected time delay. However, microlensing usually acts on the continuum rather than on the emission lines of quasar spectra, because of the much smaller size of the continuum region. Differential amplification of the continuum relative to the emission lines, as observed by Keeton et al. (2006), would be a strong support to the microlensing hypothesis. Our spectra do not show such a differential amplification, but we note that our wavelength range is very different from that of Keeton et al. (2006) and that they observed in May 2005, i.e., 3 months after our observations.

Assuming microlensing is the correct interpretation of the data, its strength depends upon the scale-size of the source, with smaller sources being more susceptible to large magnification (e.g. Wambsganss \& Paczynski 1991). The continuum emitting region and the broad-line region (BLR) of a quasar can appear small enough to undergo significant magnifications. The limiting source size for microlensing to occur is given by the Einstein radius projected onto the source plane. This means that only structures in the source with sizes comparable to or smaller than this radius will experience appreciable amplification. The Einstein radius, projected onto the source plane for microlenses with masses in the range $0.1 M_{\odot}<M<10 M_{\odot}$ is $7<R_{\mathrm{E}}<70$ lightdays for a cosmology with $\Omega_{\mathrm{m}}=0.3, \Omega_{\Lambda}=0.7$ and $h_{100}=0.65$.
Kaspi et al. (2000) derived sizes for active galaxy nuclei from reverberation mapping of the Balmer lines. As a function of intrinsic luminosity, they found a global scaling of the broad-line region (BLR) ranging from approximately 1 to 300 light days, which compares well with the Einstein radius of the microlenses in the lensing galaxy of SDSS J0924+0219.

The observations also reveal that the broad emission lines and the continuum do not vary on the same time scale. Indeed, the continuum of image A remains constant over the 15-day time span of the observations, while the broad emission lines vary.

Detailed microlensing simulations by Lewis \& Ibata (2004) show that the correlation between the magnification of the BLR and the continuum source exists, but is weak. Hence variations in the broad emission lines need not be accompanied by variations in the continuum. This argument has been confirmed through observations of other gravitationally lensed quasars (Chartas et al. 2002; Richards et al. 2004).

Another observational fact that needs some enlightening is the asymmetric amplification of the broad emission lines (see Fig. 8). Such an amplification occurs for the C II] and $\mathrm{Mg}$ II emission lines in the spectra of image A. The red wings of these lines are significantly more amplified than the blue ones. An explanation for this is given by Abajas et al. (2002) and Lewis \& Ibata (2004), who show that emission lines can be affected by substantial centroid shifts and modification of the line profile. Asymmetric modification of the line profile can be indicative of a rotating source. Microlensing of the part of the BLR that is rotating away from us would then explain the observed asymmetric line amplifications. This would imply that a microlensing caustic is passing at the edge of the broad line region, and is far enough from the continuum to leave it unaffected.

\section{HST imaging}

Optical and near-IR images of SDSS J0924+0219 are available from the HST archive in the $F 555 W, F 814 W$ and $F 160 W$ filters. The $F 555 \mathrm{~W}$ and $F 814 \mathrm{~W}$ observations have been obtained on 18 November 2003 with the Advanced Camera for Surveys (ACS) and the Wide Field Channel (WFC). The F555W data consist of two dithered $1094 \mathrm{~s}$ exposures, each one being split in two $($ CRSPLIT $=2)$ in order to remove cosmic rays. Two consecutive $1148 \mathrm{~s}$ exposures have been taken through the $F 814 \mathrm{~W}$ filter, one hour later, again splitting the exposure time in two. Finally, the NICMOS2 observations, taken on 2003 November 23, consist of 8 dithered exposures, for a total of $5312 \mathrm{~s}$. The 5-day period separating the optical and near-IR observations is of the order of the expected time delay between images A and B of the quasar.

\subsection{Image deconvolution}

The MCS algorithm (Magain et al. 1998) is used to deconvolve all images. This algorithm sharpens the images and preserves the flux of the original data. It also decomposes the data into a set of analytical point sources (the quasar images) and a numerical "extended channel" which contains all the features other than point sources, i.e., the lensing galaxy and the Einstein ring. All images are rebinned to a common pixel scale prior to deconvolution and combined with cosmic ray rejection. The reference image adopted to carry out the whole deconvolution work is the first image taken through the $F 814 \mathrm{~W}$ filter, i.e., image j8oi33031 in the HST archive. The position angle of this reference image relative to the North is $\mathrm{PA}=-2.67^{\circ}$. All the astrometry in the following is given in the coordinate system of this image. 

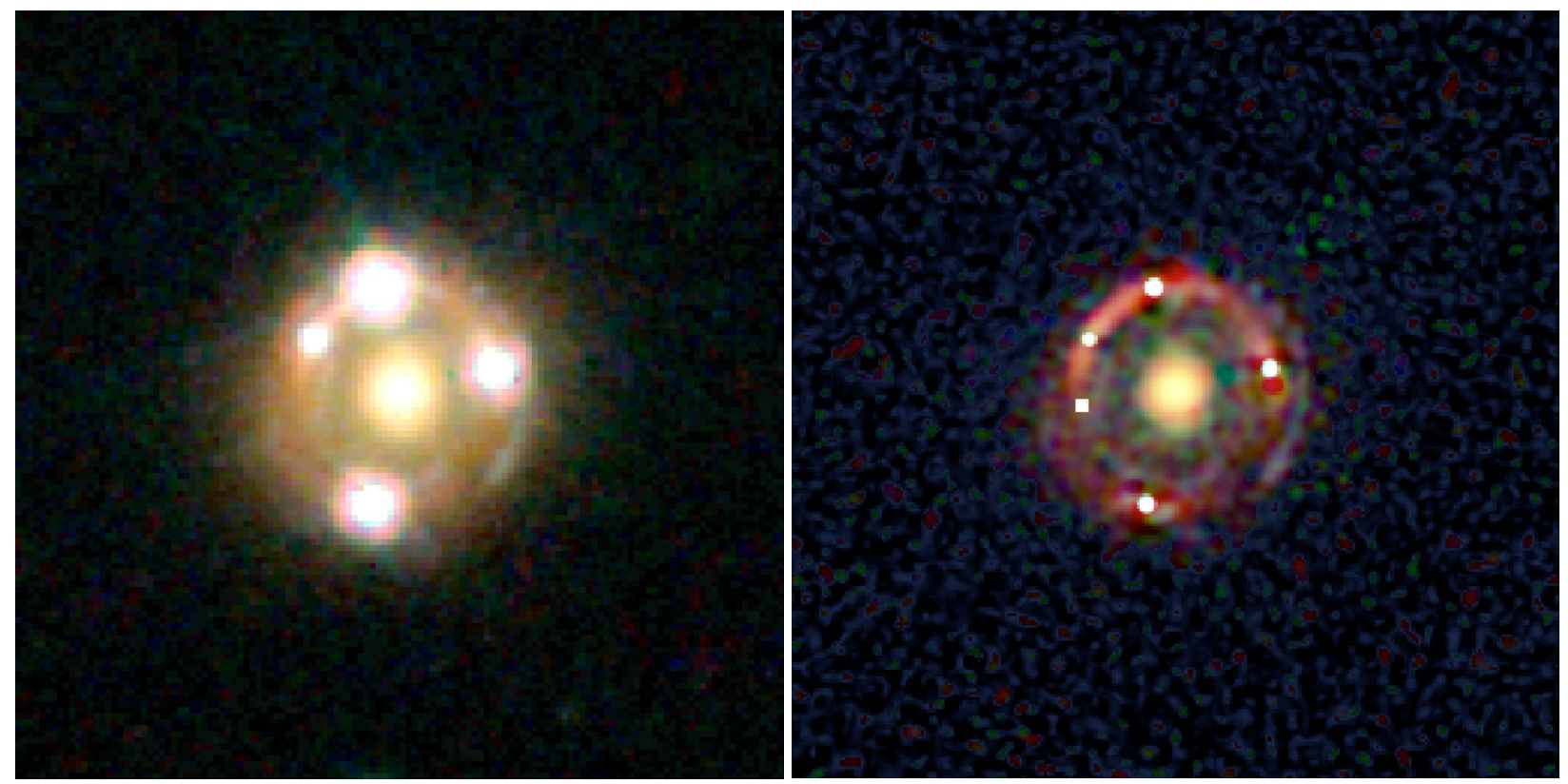

Fig. 11. Left: composite HST image using the observations through the $F 555 W, F 814 W$ and $F 160 W$ filters. The resolution is respectively $0.10^{\prime \prime}$ in $F 555 W$ and $F 814 W$, and $0.15^{\prime \prime}$ in $F 160 W$. Right: deconvolved image. It has a pixel size of $0.025^{\prime \prime}$ and a resolution of $0.05^{\prime \prime}$. The lensed host galaxy of the quasar is clearly seen as red arcs well centered on the quasar images. A second set of bluer arcs inside and outside the area delimited by the red arcs is also revealed. The field of view is $3.0^{\prime \prime}$ on a side. The image is slightly rotated relative to North, which is at PA $=-2.67^{\circ}$. East is to the left. The white square shows the position of the perturber found for the SIE and NFW models of Sect. 5.2.

The data used here are the pipeline-drizzled images available from the archive. The pixel scale in the deconvolved image is half that of the original image, i.e., $0.025^{\prime \prime} \times 0.025^{\prime \prime}$. The spatial resolution is the same in all deconvolved images, i.e., $0.05^{\prime \prime}$ Full-Width-Half-Maximum $(F W H M)$.

As the HST PSF has significant spatial variations across the field of view, stars located far away from SDSS J0924+0219 on the plane of the sky are not ideal for use in the image deconvolution. To circumvent this problem we have devised an iterative procedure. We first deconvolve the images with a fixed PSF, directly measured from stars. This gives a deconvolved image of the lens and Einstein ring, that we reconvolve with the PSF and subtract from the original data. A second PSF is re-computed from this new lens- and ring-subtracted image, directly from the quasar images, following the procedure described in Magain et al. (2005). This is similar to a blind-deconvolution, where the PSF is modified during the deconvolution process. A new deconvolved image is created with the improved PSF, as well as a new lens- and ring-subtracted image. We repeat 4 times in a row the procedure until the residual map (Magain et al. 1998; Courbin et al.1998) is flat and in average equal to $1 \sigma$ after deconvolution, i.e., until the deconvolved image becomes compatible with the data in the $\chi^{2}$ sense.

\subsection{Results}

The deconvolved images through the three filters are shown in Fig. 11, as a colour composite image. Two sets of arcs are clearly seen, corresponding to the host galaxy of the source quasar, and to a bluer object not centered on the images of the quasar. This arc is well explained by a second lensed source (see Sect. 5.2) which is either a star-forming region in the source, or another unrelated object.

Instead of using the conventional version of the MCS deconvolution algorithm, we use a version that involves a semianalytical model for the lensing galaxy. In this procedure, the
Table 2. Astrometry of SDSS J0924+0219 and flux ratio between the images. All positions are given relative to the lensing galaxy in the coordinate system of our reference HST image j8oi33031. The 1- $\sigma$ error bar on the astrometry is $0.005^{\prime \prime}$, mainly dominated by the error on the position of the lensing galaxy. The error bar on the flux ratio is of the order of $10 \%$ for images B, C and $20 \%$ for image D, and includes the systematic errors due to the presence of the Einstein ring (see text).

\begin{tabular}{lccccc}
\hline \hline Object & $\begin{array}{c}X \\
\left({ }^{\prime \prime}\right)\end{array}$ & $\begin{array}{c}Y \\
\left({ }^{\prime \prime}\right)\end{array}$ & $F 555 W$ & $F 814 W$ & $F 160 W$ \\
\hline Lens & +0.000 & +0.000 & - & - & - \\
A & -0.185 & +0.859 & 1.00 & 1.00 & 1.00 \\
B & -0.246 & -0.948 & 0.51 & 0.46 & 0.44 \\
C & +0.782 & +0.178 & 0.39 & 0.34 & 0.32 \\
D & -0.727 & +0.430 & 0.06 & 0.06 & 0.03 \\
\hline
\end{tabular}

analytical component of the lensing galaxy is either a twodimensional exponential disk, or a de Vaucouleurs profile. All slight departures from these two profiles are modeled in the form of a numerical array of pixels which includes the arcs as well.

In all bands, we find that an exponential disk fits the data much better than a de Vaucouleurs profile, which is surprising for an elliptical galaxy, as indicated by the VLT spectra. Table 2 gives a summary of our astrometry, relative to the center of the fitted exponential disk. The mean position angle of the lensing galaxy, in the orientation of the HST image, is PA $=-61.3 \pm 0.5^{\circ}$ (positive angles relative to the North in a counter-clockwise sense) and the mean ellipticity is $e=0.12 \pm 0.01$, where the error bars are the dispersions between the measurements in the three filters. We define the ellipticity as $e=1-b / a$, where $a$ and $b$ are the semi-major and semi-minor axis respectively. Note that although the formal error on the lens ellipticity and PA is small, the data show evidence for isophote twisting. The effective radius of the galaxy is $R_{\mathrm{e}}=0.50 \pm 0.05^{\prime \prime}$.

The flux ratios of the quasar images are derived from the deconvolved images. The MCS algorithm provides the user with the intensities of all point sources in the image, decontaminated 
Table 3. Best-fit parametric models for SDSS J0924+0219, obtained with the LENSMODEL package (Keeton 2001). The position angles of the lens $\theta_{\mathrm{e}}$ and of the external shear $\theta_{\gamma}$ are given in degrees, positive angles being counted counter-clockwise relative to the North. The coordinates $(x, y)$ of the centres of the models are given in arcseconds, and the time delays $\Delta t$ are expressed in days relative to the leading image $\mathrm{B}$. The extreme values for the time delays within the smallest $1-\sigma$ region of Fig. 12 are also given. We adopt a $\left(\Omega_{\mathrm{m}}, \Omega_{\Lambda}\right)=(0.3,0.7) \operatorname{cosmology}$ and $h_{100}=0.65$. All models have one degree of freedom.

\begin{tabular}{llccccccccc}
\hline \hline Model & Parameters & $(x, y)$ & $e$ & $\theta_{\mathrm{e}}$ & $\gamma$ & $\theta_{\gamma}$ & $\Delta t_{\mathrm{AB}}$ & $\Delta t_{\mathrm{CB}}$ & $\Delta t_{\mathrm{DB}}$ & $\chi^{2}$ \\
\hline \multirow{2}{*}{ SIE } & $b^{\prime}=0.87$ & $(-0.003,0.002)$ & 0.13 & -73.1 & 0.053 & 65.4 & $5.7_{5.1}^{6.7}$ & $9.1_{8.2}^{10.4}$ & $6.2_{5.5}^{7.2}$ & 0.91 \\
\multirow{2}{*}{ de Vaucouleurs } & $b=2.64$ & $(-0.004,0.002)$ & 0.16 & -70.1 & 0.096 & 77.3 & $8.6_{8.1}^{8.9}$ & $13.8_{12.9}^{14.4}$ & $9.4_{8.8}^{9.7}$ & 1.41 \\
& $R_{\mathrm{e}}=0.50$ & & & & & & & & & \\
NFW & $\kappa_{\mathrm{s}}=0.70$ & $(-0.003,0.001)$ & 0.10 & -72.0 & 0.047 & 65.4 & $4.9_{3.6}^{8.0}$ & $7.8_{5.8}^{12.7}$ & $5.4_{4.0}^{8.7}$ & 0.72 \\
& $r_{\mathrm{s}}=1.10$ & & & & & & & & & \\
\hline
\end{tabular}

from the light of the extended features, such as the ring in SDSS J0924+0219 and the lensing galaxy. The error on the quasar flux ratio is dominated by the contamination by the Einstein ring. If the intensity of a quasar image is overestimated, this will create a "hole" in the deconvolved Einstein ring at the quasar image position. If it is underestimated, the local $\chi^{2}$ at the position of the quasar image will become much larger than $1 \sigma$. The flux ratios in Table 2 are taken as the ones giving at the same time a continuous Einstein ring without any "hole", and leading to a good $\chi^{2}$, close to 1 , at the position of the quasar images. The error bars quoted in Table 2 are taken as the difference between these two extreme solutions, divided by 2 . They include both the random and systematic errors.

\section{Modeling}

Constraining the mass distribution in SDSS J0924+0219 is not trivial. Firstly, we do not have access to the true image magnifications due to contamination by microlensing and secondly, the light distribution of the lensing galaxy is not very well constrained. The ellipticity and position angle of the lens change with surface brightness, indicative of isophote twisting. Measuring the faintest isophotes on the HST data leads to $\mathrm{PA} \simeq-25^{\circ}$, as is adopted by Keeton et al. (2006) in his models. However, brighter isophotes and fitting of a PSF-deconvolved exponential disk profile yields $\mathrm{PA}=-61.3^{\circ}$.

As a blind test for the shape of the mass distribution underlying the light distribution, and without using any constraint on the ellipticity or PA of the lens, we use the non-parametric models of Saha \& Williams (2004). Fitting only the image positions returns an asymmetric lens whose major axis is aligned approximately East-West (i.e., PA $=90^{\circ}$ ). Given the discrepancy between this simple model and the observed light distribution, we test in the following a range of models with differing levels of observational constraints, in order to predict time delays.

\subsection{Parametric models}

\subsubsection{Using the flux ratios}

The LENSMODEL package (Keeton 2001) is used to carry out an analytical modeling of the lensing galaxy. Three lensing galaxy models are considered: the Singular Isothermal Ellipsoid (SIE), the Navarro et al. (1997) profile (NFW), and the de Vaucouleurs (1948) profile. In a first attempt, we constrain these models with the lensing galaxy position, the relative positions of the lensed images (Table 2) and their flux ratios (taken as the mean of the ratios measured in the three $F 555 \mathrm{~W}, \mathrm{~F} 814 \mathrm{~W}, \mathrm{~F} 160 \mathrm{~W}$ filters). If no external shear is included in the models, we find a lens ellipticity of $e \simeq 0.3$ with a PA $\theta_{\mathrm{e}} \simeq 85^{\circ}$ and an associated $\chi^{2} \simeq 200$. The ellipticity and PA agree well with the models obtained from the semi-linear inversion method of Warren \& Dye (2003) (see Sect. 5.2)

Next, we include external shear to the model. The lens position angle $\theta_{\mathrm{e}}$, coordinates, and ellipticity agree better with the measured values in the HST images. The $\chi^{2}$ values remain bad $\left(\chi^{2} \simeq 30\right)$, although improved with respect to the models without external shear. The shear orientation is $\theta_{\gamma} \sim 60^{\circ}$ which is about in the direction of a bright galaxy located $9.5^{\prime \prime}$ away from SDSS J0924+0219 and at PA $=53^{\circ}$.

The main contribution to the total $\chi^{2}$ is the anomalous flux ratios between the images of SDSS J0924+0219. In particular, the extreme flux ratio between image $\mathrm{A}$ and image $\mathrm{D}$ of $\sim 15$, when these two images are predicted to have approximately the same brightness. This is not surprising because of the evidence of microlensing in image A (Sect. 3.5) and of possible millilensing induced by massive substructures. This lead us to the considerations presented in the next section.

\subsubsection{Discarding the flux ratios}

The modeling is similar to that of Sect. 5.1.1. External shear is included but the flux ratios are discarded. In order to use only models that have one degrees of freedom (DOF), we have fixed the effective radius of the de Vaucouleurs model to the observed value. Given the number of observational constraints, the NFW model would have zero DOF if all its parameters were left free during the fit. We have therefore fixed the orientation of the external shear in this model to the value we found in the SIE+shear model. The best fit models are presented in Table 3, with (reduced) $\chi^{2}$ improved to values close to 1 .

We map the lens ellipticity vs. external shear plane in order to estimate the degree of degeneracy between these two parameters. The results are displayed in Fig. 12. It is immediatly seen that the $1-\sigma$ ellipses of the different models only marginally overlap. This is confirmed by the time delay values summarized in Table 3 where we also give the extreme values of the time delays within the $68 \%$ confidence interval. The minimum difference between the extreme time delays predicted with a constant mass-to-light ratio galaxy (de Vaucouleurs) and by the more physically plausible SIE model is about $8 \%$. Since the error measurement on the time delay propagates linearly in the error budget, even a rough estimate of the three time delays in SDSS J0924+0219, with 8\% accuracy will already allow to discriminate efficiently between flat $\mathrm{M} / \mathrm{L}$ models and SIE. Distinguishing between SIE and NFW is more difficult as time 

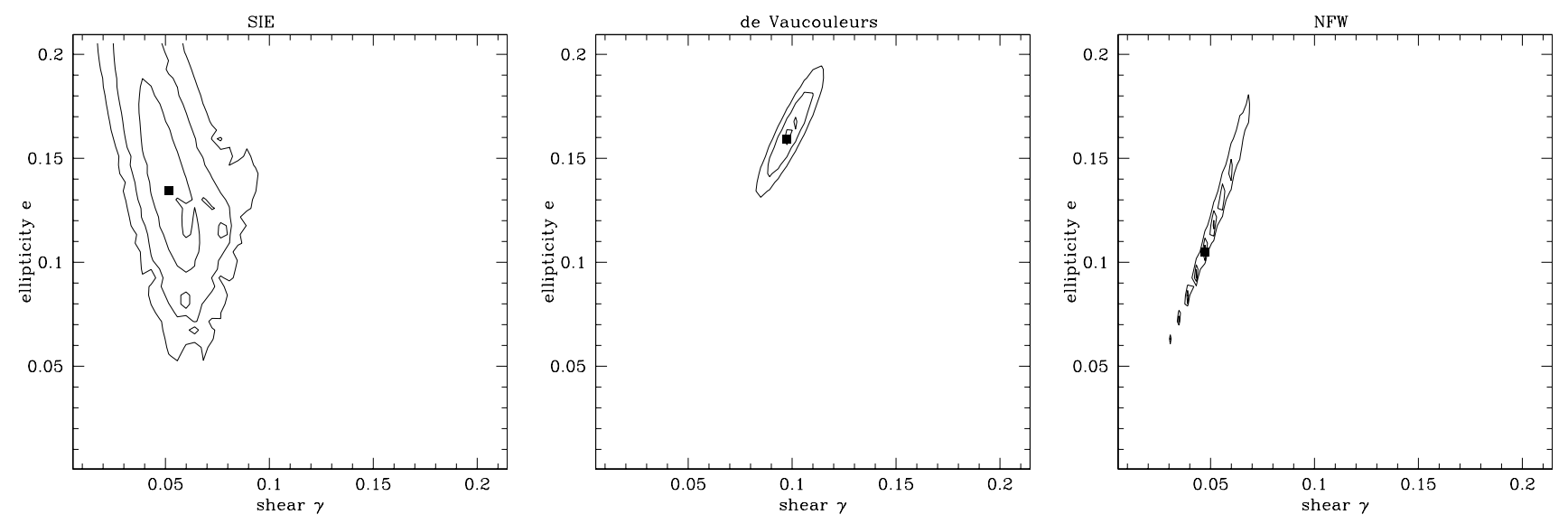

Fig. 12. The three plots give the reduced $\chi^{2}$ as a function of lens ellipticity $e$ and external shear $\gamma$ for the three analytic models used in the LENSMODEL package. No constraint is used on the image flux ratios. The contours correspond to the 1,2 and $3-\sigma$, confidence levels. The degeneracy between ellipticity and shear is clear. Only the NFW models are (marginally) compatible with no external shear at all, as also suggested by the semi-linear inversion of Sect. 5.2. The black square in each panel indicated the best fit model, which parameters are given in Table 3.

delays predicted by NFW models differ by only $1 \%$ from the SIE time delays. Such an accuracy will be hard to reach in SDSS J0924+0219, that has short time delays and a short visibility period given its equatorial position on the plane of the sky (see Eigenbrod et al. 2005).

\subsection{Using the arcs of the lensed sources}

The HST images of SDSS J0924+0219 reveal two sets of arcs. One is prominent in the near-IR (in red in Fig. 11) and is well centered on the quasar images. It is the lensed image of the quasar host galaxy. A second set of bluer arcs is best seen in $F 555 W$. It is off-centered with respect to the quasar images, indicating either a companion to the quasar host, or an independent intervening object along the line of sight.

We apply the semi-linear inversion method of Warren \& Dye (2003) to the arcs observed in the $F 555 \mathrm{~W}$ and $F 160 \mathrm{~W}$ data. The method incorporates a linear matrix inversion to obtain the source surface brightness distribution that gives the best fit to the observed lensed image for a given lens model. This linear step is carried out per trial lens parametrisation in a standard non-linear search for the global best fit.

Dye \& Warren (2005) successfully apply this technique to the Einstein ring system 0047-2808. They demonstrate that the extra constraints provided by the image of the ring results in smaller errors on the reconstructed lens model, compared to using only the centroids of the principal images as constraints in this system.

In the case of $0047-2808$, the source is a star forming galaxy without any point-like emission whereas the image of SDSS J0924+0219 is clearly dominated by the QSO's central point source. To prevent the reconstruction of SDSS J0924+0219 from being dominated by the point source and because in this section only the reconstruction of the QSO host emission is of interest, we masked out the four point source images in the $5555 \mathrm{~W}$ and $F 160 \mathrm{~W}$ data supplied to the semilinear inversion code. The astrometry of the quasar images is not used as a constraint. Figure 13 shows the masked ring images.

\subsubsection{Reconstruction results}

The deconvolved $F 160 W$ and $F 555 W$ data are reconstructed with 6 different parametric lens models. Three of these are single
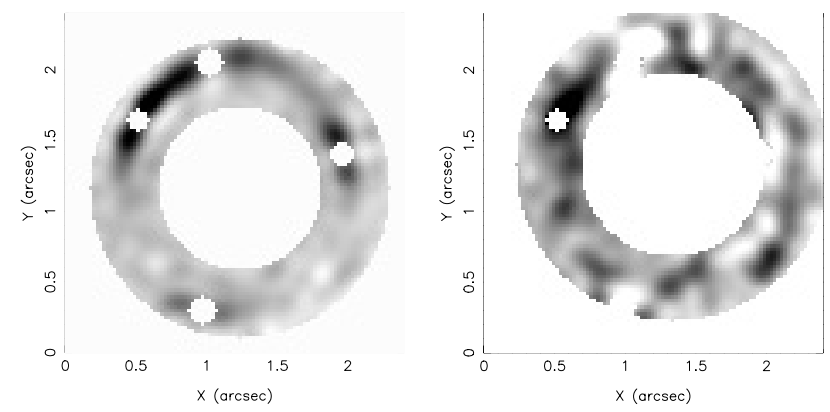

Fig. 13. Annular mask applied to the $F 160 W$ (left) and $F 555 W$ (right) data with point sources masked out. The annulus in the $F 555 \mathrm{~W}$ image is shifted by $0.1^{\prime \prime}$ to the left and $0.2^{\prime \prime}$ to the top with respect to the $F 160 \mathrm{~W}$ image, to properly encompass the blue arc seen in Fig. 11.

mass component models: the singular isothermal ellipsoid (SIE), the elliptical NFW, and the elliptical NFW with external shear. The remaining three test for asymmetry in the lens model by including a secondary singular isothermal sphere (SIS) mass component that is also free to move around in the lens plane and vary in normalization in the minimization. These models are the dual SIS model, the SIE+SIS model and the NFW+SIS model.

Since the $F 160 \mathrm{~W}$ data have the highest signal to noise arcs, we base our lens modeling on these data and applied our overall best fit model to the $F 555 \mathrm{~W}$ data to reconstruct the source. In all cases, we reconstruct with a $0.5^{\prime \prime} \times 0.5^{\prime \prime}$ source plane comprising $10 \times 10$ pixels. The reconstruction is not regularised, except in Fig. 14 where first order regularisation (see Warren \& Dye 2003) is applied to enhance visualization of the source.

Table 4 lists the minimized parameters for each model and the corresponding values of $\chi^{2}$. The SIE+SIS and NFW+SIS models clearly fare better than their single component counterparts, implying the lens is asymmetric. For the SIE+SIS, a decrease in $\chi^{2}$ of $\Delta \chi^{2}=33$ for 3 fewer degrees of freedom has a significance of $5.1 \sigma$. The decrease of $\Delta \chi^{2}=40$ for the NFW+SIS has a significance of $5.7 \sigma$. Both models consistently place the secondary SIS mass component around $\left(-0.80^{\prime \prime},-0.05^{\prime \prime}\right)$ with a normalization of only $\sim 2.5 \%$ of the main component.

Interestingly, the elliptical models listed in Table 4 have ellipticities close to those obtained with the LENSMODEL 

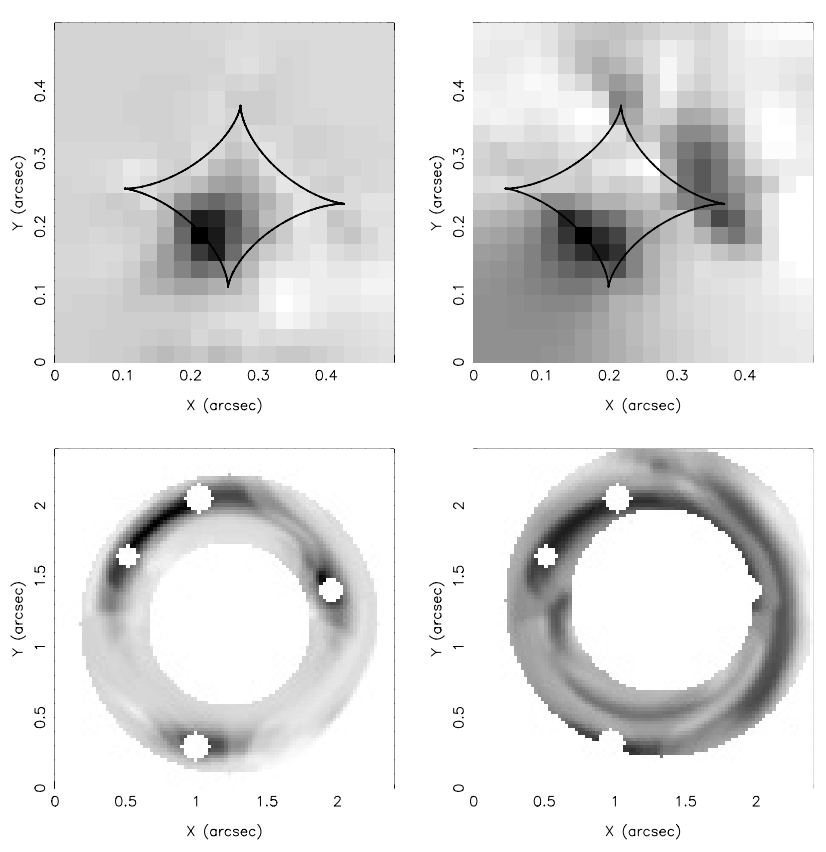

Fig. 14. Reconstructed source from $F 160 \mathrm{~W}$ data (top left) and its lensed image (bottom left). A second source lying on the rightmost cusp caustic (top right) is reconstructed from the $F 555 \mathrm{~W}$ image corresponding to the blue arc (bottom right).

software, when no external shear is considered. When external shear is added to the NFW model, we do indeed obtain a significantly better fit compared to the NFW on its own, but the results differ from those listed in Table 3. While the ellipticity remains almost the same as in Table 3, its PA differs by approximately $25^{\circ}$. Moreover, we find a ten times smaller amplitude for the shear using the semi-linear inversion than using LENSMODEL. Note, however, that the observed quasar image astrometry is used in the LENSMODEL analysis, whereas it is not in the present semi-linear inversion. If we use the lens model found by the semi-linear inversion to predict the position of the quasar images, we find poor agreement between the predicted and the measured positions. The global, large scale shape of the lens found by the semi-linear inversion is well adapted to model the Einstein rings, which are very sensitive to azimuthal asymmetry in the lens, but additional smaller scale structures are needed to slightly modify the positions of the quasar images and make them compatible with the measured astrometry. The disagreement between the astrometry predicted by LENSMODEL and the one predicted by the semi-linear inversion adds support to the presence of multipole-type substructures in the lens (e.g., Congdon \& Keeton 2005).

The top left plot in Fig. 14 shows the reconstructed source corresponding to the best fit NFW+SIS model for the F160W data. The observed arcs are explained by a single QSO host galaxy. Note that in this figure, purely to aid visualization, we have regularised the solution and plotted the surface brightness with a pixel scale half that used in the quantitative reconstruction. The bottom left corner of Fig. 14 shows the image of the reconstructed source lensed by the best fit NFW+SIS model.

We then take the best fit NFW+SIS model in order to reconstruct the $F 555 W$ data shown on the right in Fig. 13. Note that the annular mask is shifted slightly compared to the $F 160 \mathrm{~W}$ data, to properly encompass the blue arc. The reconstructed source and corresponding lensed image are shown on the right hand side of Fig. 14.
There are two distinct sources now visible. The QSO host identified previously has again been reconstructed. This is because its dominant image, the bright arc in the top left quadrant of the ring, is still present in the $F 555 W$ data. A second source, more diffuse and lying on the rightmost cusp caustic is also visible. This second source is responsible for the blue arcs.

The redshift of the second source remains unknown. It could be a star forming object/region lying $0.2^{\prime \prime} \cdot D_{\mathrm{s}} \simeq 1200 h_{100}^{-1} \mathrm{pc}$ away from the quasar, i.e., it would be part of the host galaxy. It is, however, not excluded that this second source is at a different redshift than the quasar, e.g. located between the quasar and the lens, as it is bluer than the quasar host galaxy. If the latter is true, SDSS J0924+0219 might be a unique object to break the mass sheet degeneracy. Unfortunately, the lens modeling alone, does not allow to infer a redshift estimate.

\subsection{Note on the different types of models}

The two methods used above differ in several respects. LENSMODEL has a limited number of free parameters but uses only the constraints on the astrometry of the quasar images. While a qualitative representation of the lensed host galaxy of the quasar source can be attempted, the method does not allow a genuine fitting of the Einstein rings assuming a (simplified) shape for the quasar host.

The semi-linear inversion carries out a direct reconstruction of the lensed source as a whole, where each pixel of the HST image is a free parameter. As the quasar images largely dominate the total flux of the source, they need to be masked before the reconstruction. For this reason it is not possible with this method, at the present stage of its development, to constrain the lens model using simultaneously the astrometry of the quasar images and the detailed shape of the Einstein rings.

Although the two methods used in the present work are fundamentally different and although they use very different observational constraints, they agree on the necessity to bring extra mass near image D of SDSS J0924+0219. Smooth lenses like the ones implemented in LENSMODEL have PAs that differ by $10^{\circ}$ from the one measured in the HST images. In the orientation of Fig. 11, the mass distribution found by LENSMODEL is closer to horizontal $\left(\mathrm{PA}=-90^{\circ}\right)$ than the light distribution, hence giving larger masses next to image D. In the semi linear inversion, the optimal position found for the SIS perturber is also close to image D.

Given the above discussion, the poor determination of the lens PA is a main limitation to the interpretation of the time delays in SDSS J0924+0219. An alternative route is to determine the dynamical rotation axis of the lens, a challenge which is now within the reach of integral field spectroscopy with large telescopes and adaptive optics.

\section{Conclusions}

We have spatially deconvolved deep sharp VLT/FORS1 MOS spectra of SDSS J0924+0219, and measured the redshift of the lensing galaxy, $z_{\text {lens }}=0.394 \pm 0.001$, from numerous stellar absorption lines. The spectrum beautifully matches the elliptical galaxy template of Kinney et al. (1996).

The flux ratio between image $\mathrm{A}$ and $\mathrm{B}$ is $F_{\mathrm{A}} / F_{\mathrm{B}}=2.80 \pm$ 0.05 on 2005 January 14 , and $F_{\mathrm{A}} / F_{\mathrm{B}}=2.86 \pm 0.05$ on 2005 February 1, i.e., it has not changed between the two dates given the uncertainties on the flux ratios (Table 1). For each date, this ratio is mostly the same in the continuum and in the broad 
Table 4. Minimized lens model parameters and corresponding $\chi^{2}$. Model parameters are: $\kappa_{0}=$ mass normalization in arbitrary units, $(x, y)=$ offset of lens model centre from lens optical axis in arcseconds, $e=$ ellipticity, $\gamma=$ external shear, $\theta_{\mathrm{e}}$ and $\theta_{\gamma}=$ PA in degrees counted counter-clockwise from North. In the case of the NFW, the scale radius is held fixed at 6" in the minimization. The third column gives the number of degrees of freedom (NDOF). Subscript " $b$ " refers to the secondary SIS in the dual component models (see text).

\begin{tabular}{lccl}
\hline \hline Model & $\chi_{\min }^{2}$ & NDOF & Minimized parameters \\
\hline SIE & 4280 & 3975 & $\kappa_{0}=100.0,(x, y)=(0.02,0.04), e=0.270, \theta_{\mathrm{e}}=86.0$ \\
NFW & 4011 & 3974 & $\kappa_{0}=100.0,(x, y)=(0.06,0.06), e=0.187, \theta_{\mathrm{e}}=84.9$ \\
Dual SIS & 4385 & 3974 & $\kappa_{0}=49.2,(x, y)=(0.00,0.28), \kappa_{0 b}=51.6,(x, y)_{b}=(-0.06,-0.33)$ \\
SIE+SIS & 4247 & 3972 & $\kappa_{0}=99.4,(x, y)=(0.04,0.04), e=0.265, \theta_{\mathrm{e}}=85.1, \kappa_{0 b}=2.1,(x, y)_{b}=(-0.79,-0.03)$ \\
NFW+SIS & 3971 & 3971 & $\kappa_{0}=98.0,(x, y)=(0.05,0.08), e=0.206, \theta_{\mathrm{e}}=83.1, \kappa_{0 b}=2.8,(x, y)_{b}=(-0.80,-0.09)$ \\
NFW $+\gamma$ & 3992 & 3972 & $\kappa_{0}=100.0,(x, y)=(0.06,0.06), e=0.168, \theta_{\mathrm{e}}=86.0, \gamma=0.010, \theta_{\gamma}=78.3$ \\
\hline
\end{tabular}

emission lines of the quasar images $\mathrm{A}$ and $\mathrm{B}$. This may seem in contradiction with Keeton et al. (2006) who see differential amplification of the continuum relative to the lines, but our observing dates and setup are very different from theirs.

While the continuum of images A and B has not changed in 15 days, there are obvious and asymmetric changes in some of the quasar broad emission lines. Microlensing of both A and B is compatible with this, although somewhat ad hoc assumptions must be done on the position of the microcaustics relative to the quasar, as well as on the relative sizes of the continuum and broad line regions.

Deep HST imaging reveals two sets of arcs. One corresponds to the red lensed host galaxy of the quasar and defined an Einstein ring connecting the quasar images. The other, fainter and bluer is off-centered with respect to the quasar images. It is either a star-forming region in the quasar source host galaxy, or another intervening object.

The lens ellipticity and PA measured in the HST images are hard to reconcile with simple models without external shear. The model fits improve when external shear is added, even though the predicted PA differs from the measured one by approximately $25^{\circ}$.

Models of Sect. 5.2, involving an additional small (SIS) structure to the main lens always place it along the East-West axis, about $0.8^{\prime \prime}$ to the East of the main lens, i.e., towards the demagnified image $\mathrm{D}$. In addition, the models reconstructed using only the Einstein rings do not predict the correct astrometry for the quasar images. Einstein rings constrain the overall, large scale of the lens. Small deviations from this large scale shape are needed to match the quasar images astrometry. The discrepancy between the models using the rings and the ones using the quasar image positions therefore adds support to the presence of multipole-like substructures in the lens of SDSS J0924+0219.

Finally, the range of time delays predicted by the different lens models is large and is very sensitive to the presence of external shear and to the determination of the main lens ellipticity and PA. The time delay measurement and the lens modeling, combined with integral field spectroscopy of the lens in SDSS J0924+0219 might therefore prove extremely useful to map the mass-to-light ratio in the lens, by comparing the lensing and dynamical masses, to the light distribution infered from the HST images.

Acknowledgements. The authors would like to thanks Dr. Steve Warren for useful discussions and the ESO staff at Paranal for the care taken with the crucial slit alignment necessary to carry out the spectra deconvolutions. The HST archive data used in this article were obtained in the framework of the CfA-Arizona Space Telescope LEns Survey (CASTLES, HST-GO-9744, PI: C. S. Kochanek). P.M. acknowledges support from the PSS Science Policy (Belgium) and by PRODEX (ESA). COSMOGRAIL is financially supported by the Swiss National Science Foundation (SNSF).

\section{References}

Abajas, C., Mediavilla, E., Muñoz, J. A., et al. 2002, ApJ, 576, 640 Chartas, G., Agol, E., Eracleous, M., et al. 2002, ApJ, 658, 509 Congdon, A. B., \& Keeton, C. R. 2005, MNRAS, 364, 1459

Courbin, F., Lidman, C., Frye, B. L., et al. 1998, ApJ, 499, L199 Courbin, F., Magain, P., Kirkove, M., et al. 2000, ApJ, 529, 1136 Courbin, F., Saha, P., \& Schechter, P. L. 2002, Lecture Note in Physics, ed. Courbin, \& Minniti (Springer Verlag: Berlin), 608, 1

de Vaucouleurs, G. 1948, Ann. Astrophys., 11, 247

Dye, S., \& Warren, S. J. 2005, ApJ, 623, 31

Eigenbrod, A., Courbin, F., Vuissoz, C., et al. 2005, A\&A, 436, 25

Faber, S. M., Tremaine, S., Ajhar, E. A., et al. 1997, AJ, 114, 1771

Hernquist, L. 1990, ApJ, 356, 359

Inada, N., Becker, R. H., Burles, S., et al. 2003, AJ, 126, 666

Jaffe, W. 1983, MNRAS, 202, 995

Kaspi, S., Smith, P. S., Netzer, H., et al. 2000, ApJ, 533, 631

Keeton, C. R. 2001 [arXiv: astro-ph/0102340]

Keeton, C. R., Burles, S., Schechter, P. L., \& Wambsganss, J. 2006, ApJ, 639, 1

Kinney, A. L., Calzetti, D., Bohlin, R. C., et al. 1996, ApJ, 467, 38

Kochanek, C. S. 2005a, in Proceedings of the 33rd Saas Fee advanced course, Gravitational Lensing: Strong, Weak \& Micro, ed. G. Meylan, P. Jetzer, \& P. North (Springer Verlag: Berlin)

Kochanek, C. S. 2005b, in Impact of Gravitational Lensing on Cosmology, ed. Y. Mellier, \& G. Meylan, IAU Symp., 225, 205 [arXiv: astro-ph/0412089]

Kochanek, C. S., \& Dalal, N. 2004, ApJ, 610, 69

Lewis, G. F., \& Ibata, R. A. 2004, MNRAS, 348, 24

Magain, P., Courbin, F., \& Sohy, S. 1998, ApJ, 494452

Magain, P., Courbin, F., Sohy, S., \& Gillon, M. 2005, in preparation

Moore, B., Governato, F., Quinn, T., et al. 1998, ApJ, 499, L5

Muñoz, J. A., Kochanek, C. S., \& Keeton, C. R. 2001, ApJ, 558, 657

Navarro, J. F., Frenk, C. S., \& White, S. D. M. 1997, ApJ, 490, 493

Ofek, E. O., Maoz, D., Rix, H.-W., et al. 2005 [arXiv: astro-ph/0510465]

Refsdal, S. 1964, MNRAS, 128, 307

Richards, G. T., Keeton, C. R., Bartosz, P., et al. 2004, ApJ, 610, 679

Saha, P., \& Williams, L. L. R. 2004, AJ, 127, 2604

Schechter, P. L., Udalski, A., Szymanski, M., et al. 2003, ApJ, 584, 657

Schechter, P. L., Wambsganss, J., \& Lewis, G. F. 2004, ApJ, 613, 77

Wambsganss, J., \& Paczynski, B. 1991, AJ, 102, 864

Warren, S. J., \& Dye, S. 2003, ApJ, 590, 673 